\title{
Characteristics of female lung cancer in Korea: analysis of Korean National Lung Cancer Registry
}

Jeong Uk Lim ${ }^{1}$, Solji Han ${ }^{2}$, Ho Cheol Kim ${ }^{3}$, Chang Min Choi ${ }^{3}$, Chi Young Jung ${ }^{4}$, Deog Gon Cho ${ }^{5}$, Jae Hyun Jeon ${ }^{6}$, Jeong Eun Lee ${ }^{7}$, Jin Seok Ahn ${ }^{8}$, Yeongdae Kim ${ }^{9}$, Yoo-Duk Choi ${ }^{10}$, Yang-Gun Suh ${ }^{11}$, Jung-Eun Kim ${ }^{12}$, Young-Joo Won ${ }^{12}$, Young-Chul Kim ${ }^{13}$, Chan Kwon Park ${ }^{1 \#}$, Seung Joon Kim ${ }^{14,15 \#}$

${ }^{1}$ Division of Pulmonary, Allergy and Critical Care Medicine, Department of Internal Medicine, Yeouido St. Mary's Hospital, College of Medicine, The Catholic University of Korea, Seoul, Korea; ${ }^{2}$ Department of Applied Statistics, Yonsei University, Seoul, Korea; ${ }^{3}$ Department of Pulmonary and Critical Care Medicine, Asan Medical Center, College of Medicine, University of Ulsan, Seoul, Korea; ${ }^{4}$ Department of Internal Medicine, Daegu Catholic University School of Medicine, Daegu, Korea; ${ }^{5}$ Department of Thoracic \& Cardiovascular Surgery, St. Vincent's Hospital, College of Medicine, The Catholic University of Korea, Suwon, Korea; ${ }^{6}$ Department of Thoracic and Cardiovascular Surgery, Seoul National University Bundang Hospital, Seoul National University College of Medicine, Seoul, Korea; ${ }^{7}$ Division of Pulmonology Department of Internal Medicine, Chungnam National University, Daejeon, Korea; ${ }^{8}$ Department of Medicine, Samsung Medical Center, Sungkyunkwan University, Seoul, Korea; ${ }^{9}$ Department of Cardiothoracic Surgery, Pusan National University Hospital, Pusan, Korea; ${ }^{10}$ Department of Pathology, Chonnam National University, Hwasun Hospital, Hwasun, Korea; ${ }^{11}$ Proton Therapy Center, Research Institute and Hospital, National Cancer Center, Goyang, Korea; ${ }^{12}$ Cancer Registration and Statistics Branch, National Cancer Center, Goyang, Korea; ${ }^{13}$ Department of Internal Medicine, Chonnam National University, Hwasun Hospital, Hwasun, Korea; ${ }^{14}$ Division of Pulmonary, Allergy and Critical Care Medicine, Department of Internal Medicine, Seoul St. Mary's Hospital, College of Medicine, The Catholic University of Korea, Seoul, Korea; ${ }^{15}$ Postech-Catholic Biomedical Engineering Institute, College of Medicine, The Catholic University of Korea, Seoul, Korea

Contributions: (I) Conception and design: JU Lim, SJ Kim; (II) Administrative support: CM Choi, YD Kim, JE Kim, YJ Won, YC Kim; (III) Provision of study materials or patients: HC Kim, CM Choi, CY Jung, DG Cho, JH Jeon, JE Lee, JS Ahn, YD Kim, YD Choi, YG Suh, CK Park; (IV) Collection and assembly of data: JE Kim, YJ Won, YC Kim; (V) Data analysis and interpretation: JU Lim, S Han, SJ Kim; (VI) Manuscript writing: All authors; (VII) Final approval of manuscript: All authors.

"These authors contributed equally to this work.

Correspondence to: Chan Kwon Park, MD, PhD. Division of Pulmonology and Critical Care Medicine, Department of Internal Medicine, Yeouido St. Mary`s Hospital, College of Medicine, The Catholic University of Korea, \#62 Yeouido-dong, Yeongdeungpo-gu, Seoul 150-713, Korea. Email: ckpaul@catholic.ac.kr; Seung Joon Kim, MD, PhD. Division of Pulmonology, Department of Internal Medicine, Seoul St. Mary's Hospital, College of Medicine, The Catholic University of Korea, 222, Banpo-daero, Seocho-gu, Seoul 06591, Korea. Email: cmcksj@catholic.ac.kr.

Backgrounds: The present study evaluated Korean women with lung cancer and compared the clinical characteristics of ever-smoker and never-smoker groups using the National Lung Cancer Registry.

Methods: In affiliation with the Korean Central Cancer Registry, the Korean Association for Lung Cancer constructed a registry into which $10 \%$ of the lung cancer cases in Korea were registered. Female lung cancer patients with valid smoking history were evaluated.

Results: Among 735 female lung cancer patients, 643 (87.5\%) were never-smokers and 92 (12.5\%) were smokers. The median survival was significantly longer in the never-smoker group (28 vs. 14 months; $\mathrm{P}<0.001)$. Among 683 patients with non-small cell lung cancer (NSCLC), the never-smoker group showed significantly longer median survival (29 vs. 14 months; $\mathrm{P}=0.002)$ and a higher proportion of stage I cancer (40.3\% vs. $25.7 \%$; $\mathrm{P}<0.001$ ). Survival analysis of the NSCLC patients showed that smoking status, receiving only supportive care, EGFR mutation status, lung cancer stage, and forced vital capacity (FVC) (\%) were significantly associated with mortality in the multivariate analysis $(\mathrm{P}=0.025, \mathrm{HR} 2.39,95 \%$ CI: 1.12-5.11; $\mathrm{P}=0.017$, HR 3.14, 95\% CI: 1.22-8.06; $\mathrm{P}=0.033$, HR 0.63, 95\% CI: 0.41-0.96; $\mathrm{P}<0.001$, HR 11.88, 95\% CI: 5.79-24.38; P=0.002, HR 0.98, 95\% CI: 0.96-0.99, respectively).

Conclusions: In Korean women with NSCLC, smoking status, not receiving active anticancer treatment, EGFR mutation status, lung cancer stage, and pulmonary function were significantly associated with mortality.

Keywords: Women; lung cancer; smoking; pulmonary function; mortality 
Submitted Apr 15, 2020. Accepted for publication Aug 12, 2020.

doi: $10.21037 /$ jtd-20-1671

View this article at: http://dx.doi.org/10.21037/jtd-20-1671

\section{Introduction}

Lung cancer is a leading cause of cancer-related mortality worldwide $(1,2)$. In the United States, lung cancer is one of the most common cancers and the leading cause of cancerrelated mortality in women (3). In other countries, increases in lung cancer mortality have been observed in parallel with the prevalence of cigarette smoking (4). Lung cancer in women shows different clinical characteristics compared to men regarding pathophysiology, prognosis, and related risks (5-7). The differences may be due to the environment, hormones, and other factors $(8,9)$, and lung cancer in females could be a distinct disease entity.

However, the clinical presentation of lung cancer in women can also differ depending on region and race. Asian women with lung cancer have distinct clinical characteristics compared to women in Western populations. The prevalence of smoking in women with lung cancer is less than $20 \%$ in Asian regions $(10,11)$, whereas $70-85 \%$ of women with lung cancer in Western populations, including North America, northern Europe, and Australia/New Zealand, were reported to be smokers (12). In addition, it is widely known that the prevalence of epidermal growth factor receptor (EGFR) mutation is higher in Asian females than in Western populations $(13,14)$.

The proportion of women in the lung cancer population has been increasing in Korea (15). Among women, we can speculate that the clinical characteristics of smokers would be less favorable compared to never-smokers among lung cancer patients. They would have poorer lung functions and the prevalence of EGFR mutation would be lower $(13,16,17)$. Thus, it is also important to evaluate how smoking affects the prognosis of women with lung cancer. In this context, the real-life data of female lung cancer patients would provide useful clinical information.

In affiliation with the Korean Central Cancer Registry (KCCR), the Korean Association for Lung Cancer (KALC) built a registry (KALC-R) into which $10 \%$ of the lung cancer cases from all Korean nationals were registered after the survey of the patients. This registry was developed to construct an unbiased lung cancer database to represent the Korean lung cancer population.

Using data from the KALC-R for 2014, the present study evaluated Korean women with lung cancer and further compared the clinical characteristics of ever-smoker and never-smoker groups. We present the following article in accordance with the STROBE reporting checklist (available at http://dx.doi.org/10.21037/jtd-20-1671).

\section{Methods}

\section{Study patient selection}

In 2014, 24,253 patients with newly diagnosed lung cancer were registered in the KCCR. After excluding patients who were enrolled in more than one institution or did not fit the KCCR enrollment criteria, 21,960 patients from 13 regional cancer centers and 39 hospitals remained as the final study population. Based on the annual number of patients registered in each hospital, the sample size allocated to each hospital was decided after taking selection probability into account. After sorting patients by age, sex, date of the first diagnosis, and the Surveillance, Epidemiology, and End Results program (SEER) summary stage, 2,640 were selected from 52 hospitals using a systematic sampling method (18). After excluding 19 patients with multiple primary cancers, 745 female lung cancer patients were selected from the remaining 2,621 patients. Thus, after exclusion of male patients and those without valid smoking history, 735 female lung cancer patients with valid smoking history were evaluated retrospectively in the present study. The selection process is shown in Figure 1.

\section{Data collection}

Using a standardized protocol, patient data including age; sex; treatment modalities; smoking status; histopathologic type; initial symptoms; Eastern Corporative Oncology Group (ECOG) score; cancer stage (according to the seventh edition of the TNM International Staging System); driver mutations, such as EGFR mutations and anaplastic lymphoma kinase (ALK) translocations; body mass index (BMI); and survival status were collected. Furthermore, forced expiratory volume in $1 \mathrm{~s}$ (FEV1), forced vital capacity (FVC), and FEV1/FVC were also collected in the study. Choi's reference equation, the reference equation for the Korean population, was used to calculate the predicted $\mathrm{FEV} 1 \%$ and predicted $\mathrm{FVC} \%$ in the study 


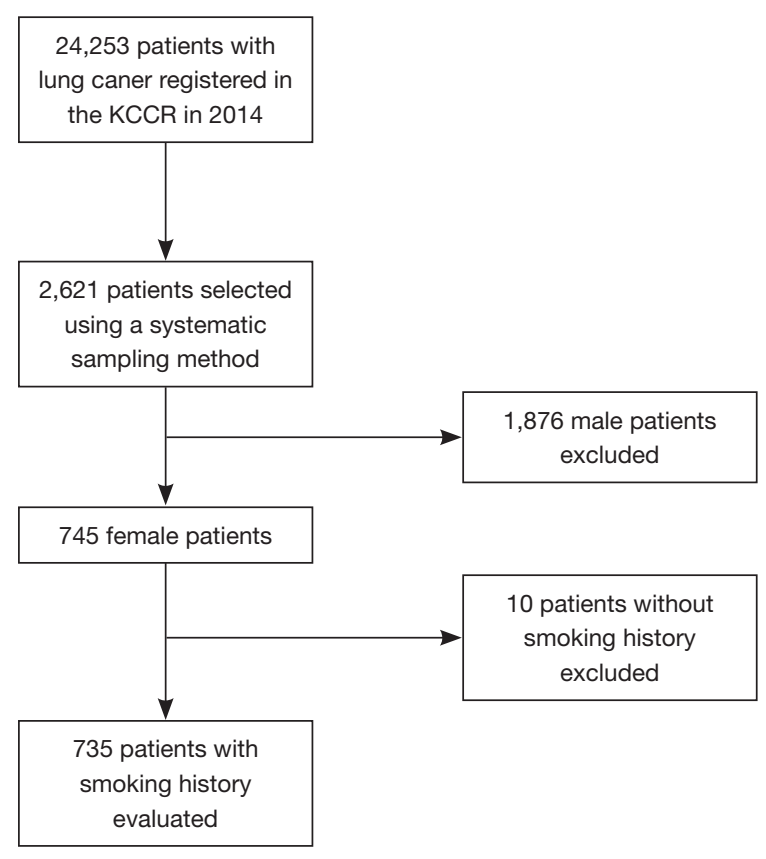

Figure 1 Selection process of the study patients.

patients (19). Regarding smoking status, ever-smokers were defined as patients with any history of smoking before a lung cancer diagnosis and never-smokers as those with no smoking history. The patients were followed-up until December 2017.

\section{Statistical analysis}

To compare the demographic and clinical characteristics between the two groups, we used the two-sample $t$-test for continuous variables and the chi-squared test for categorical variables. The overall survival (OS) of the patients was estimated from Kaplan-Meier survival curves and the statistical difference between the groups was tested by the log-rank test. OS was defined as the time from the lung cancer diagnosis to the date of death or the date of the last follow-up.

The Cox proportional hazard model was used to identify independent prognostic factors in the study and variables that were statistically significant in the univariate analysis were entered into multivariate analysis. Hazard ratios (HRs) and $95 \%$ confidence intervals (95\% CIs) were estimated. A $\mathrm{P}$ value of $<0.05$ was considered statistically significant for all tests. Statistical analyses were performed using R (version 3.5.1; R Computing, Vienna, Austria).

\section{Ethical statement}

The study was conducted in accordance with the Declaration of Helsinki (as revised in 2013). The study protocol was reviewed and approved by the Institutional Review Board at the National Cancer Center (NCC20180193), which waived the requirement for informed consent due to the retrospective nature of the study.

\section{Results}

\section{Patients clinical characteristics}

A total of 735 female lung cancer patients were selected and evaluated. There were $643(87.5 \%)$ never-smokers and 92 (12.5\%) smokers. Among 92 ever smokers, 61 (66.3\%) were current smoker and 31 (33.7\%) were former smokers. Mean pack years of the smoker group was $23.8 \pm 20.6$. The clinical characteristics were compared between the two groups and are shown in Table 1. There was no significant difference in age and initial symptoms between the two groups. The never-smoker group had significantly higher BMIs than the smoker group (23.8 vs. 22.8; $\mathrm{P}=0.023$ ), and had a higher proportion of patients with good performance (ECOG 0-2) with statistical significance (96\% vs. 88.6\% in the never-smokers $v s$. smokers, respectively; $\mathrm{P}=0.017$ ). In addition, the never-smoker group showed significantly better lung function than the smoker group. The proportion of adenocarcinoma was significantly higher in the never-smoker group. Moreover, the median survival was significantly higher in the never-smoker group (28 vs. 14 months; $\mathrm{P}<0.001$ ).

\section{Comparison of NSCLC in the never-and ever-smoker groups}

Among 735 patients, a total of 683 patients were categorized as NSCLC (92.9\%). There were $613(89.8 \%)$ neversmokers and 70 ever-smokers (10.2\%). Mean pack years of the smoker group was $22.1 \pm 18.7$. The never-smoker NSCLC patients showed significantly higher BMIs (25.1 vs. $22.5 ; \mathrm{P}=0.017)$ and were significantly younger $(66$ vs. 69; $\mathrm{P}=0.048)$. No statistical differences were found between the initial symptoms and ECOG scores. The never-smoker NSCLC patients showed significantly longer median survival (29 vs. 14 months; $\mathrm{P}=0.002$ ) (Figure 2), and a higher proportion of stage I cancer (40.3\% vs. $25.7 \%$; $\mathrm{P}<0.001)$. However, no significant difference was seen in 
Table 1 Baseline clinical characteristics of the study patients

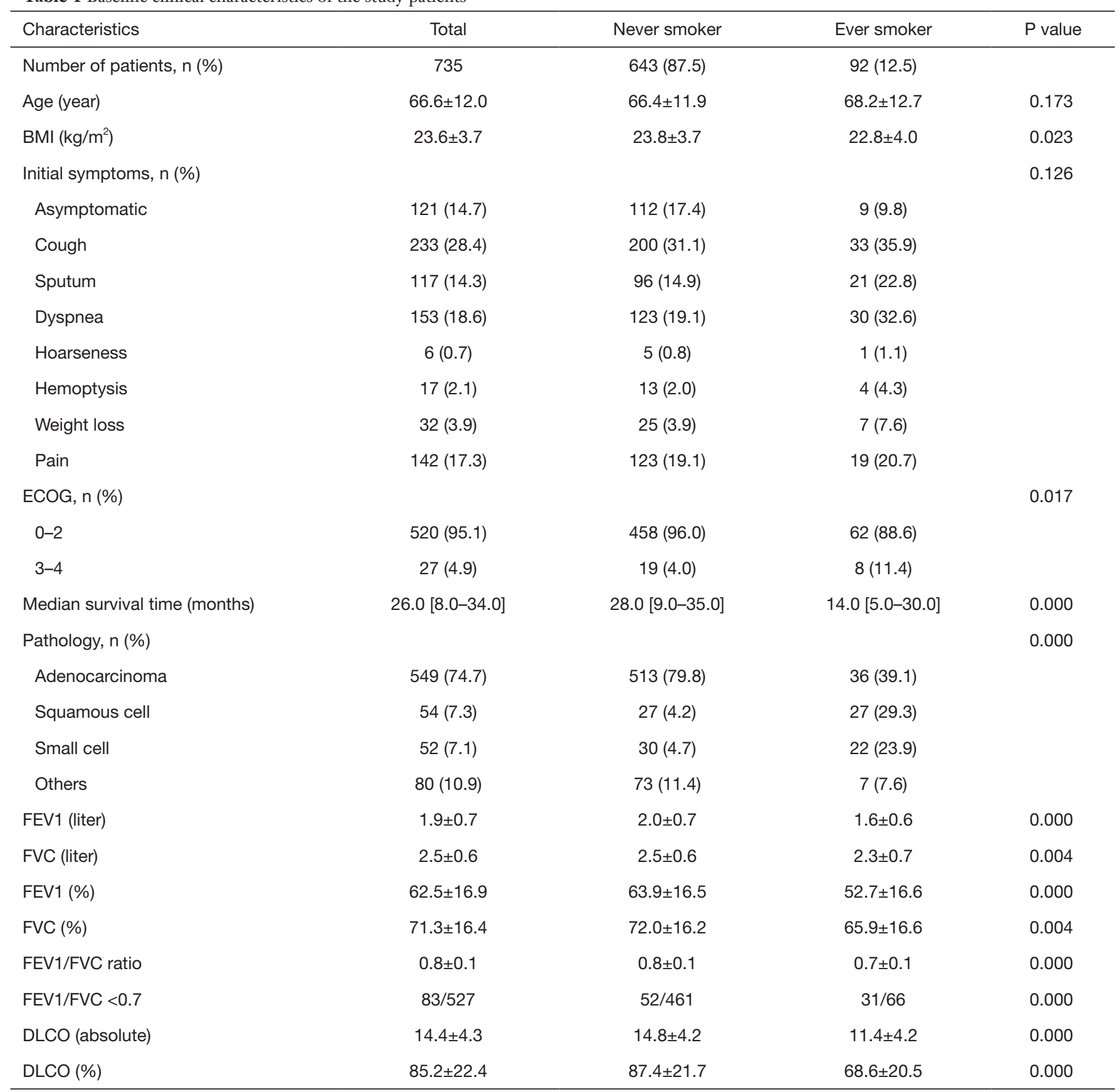

BMI, body mass index; ECOG, Eastern Cooperative Oncology Group; FEV1, forced expiratory volume in 1 s; FVC, forced vital capacity; DLCO, diffusing capacity of the lung for carbon monoxide.

the prevalence of EGFR or ALK mutations. The treatment modalities and parameters related to pulmonary function were also compared between the two groups and are shown in Table 2.

\section{Stage IV NSCLC}

From 683 patients with NSCLC, 298 patients were categorized with stage IV cancer. There was no statistically 
Kaplan-Meier survival estimates of the patients in NSCLC

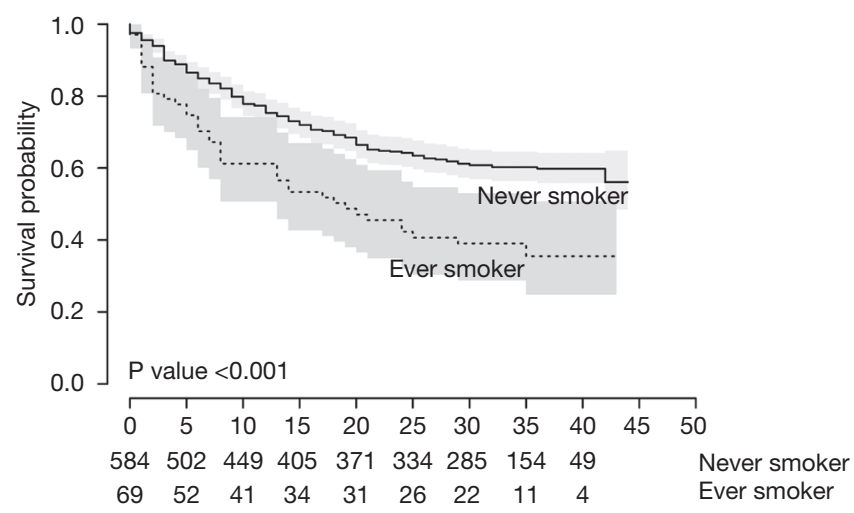

Figure 2 Kaplan-Meier survival curves for the ever and never smoker patients with non-small cell lung cancer.

significant difference between the two groups with respect to age, BMI, initial symptoms, ECOG, primary mass size or the proportion of driver mutations (EGFR and ALK). A significantly higher proportion of never-smoker patients received targeted therapy as an initial treatment compared to ever-smoker patients ( $28 \%$ vs. $8 \% ; \mathrm{P}=0.022)$, whereas diffusing capacity for carbon monoxide (DLCO) (\%) was lower and the proportion of patients with FEV1/FVC $<0.7$ was higher in the smoker group with statistical significance $(\mathrm{P}=0.035$ and $\mathrm{P}=0.028$, respectively).

\section{Comparison between EGFR mutation-positive and wild- type NSCLC groups}

Among 481 patients who underwent EGFR tests, 248 (51.6\%) were EGFR wild-type and 233 (48.4\%) were EGFR mutation-positive. There was no significant difference between the two groups in BMI, age, smoking status, ECOG or pulmonary function parameters. The EGFR mutation group showed significantly longer survival time (29.0 vs. 23.5 months; $\mathrm{P}=0.001$ ) (Figure 3), and a higher proportion of stage I cancer $(39.8 \%$ and $29.7 \% ; \mathrm{P}=0.009)$. In addition, a higher proportion of the EGFR mutation group underwent targeted therapy as an initial treatment (30.3\% vs. $1.7 \%$ in EGFR mutation-positive vs. EGFR wild-type, respectively; $\mathrm{P}<0.001$ ) (Table S1).

\section{Survival analysis in NSCLC}

Table 3 shows both univariate and multivariate survival analyses in all NSCLC patients. Smoking status, receiving only supportive care for treatment, EGFR mutation status, lung cancer stage, and FVC (\%) showed significant associations with mortality in multivariate analysis $(\mathrm{P}=0.025$, HR 2.39, 95\% CI: 1.12-5.11; $\mathrm{P}=0.017$, HR 3.14, 95\% CI: 1.22-8.06; $\mathrm{P}=0.033$, HR 0.63, 95\% CI: 0.41-0.96; $\mathrm{P}<0.001$, HR 11.88, 95\% CI: 5.79-24.38; P=0.002, HR 0.98, 95\% CI: 0.96-0.99, respectively).

\section{Discussion}

The present study described the clinical characteristics of female lung cancer patients in Korea and focused on comparing the clinical presentation between ever-smoker and never-smoker groups. The strength of this study was the use of national cancer registry data, which are data representing all Korean patients with lung cancer.

Asian women with lung cancer show distinct clinical characteristics compared to European populations. First, the proportion of ever-smokers is low compared to Western populations (12). A previous study of Korean lung cancer patients showed that the proportion of ever-smokers was about $10 \%$ (10). In contrast, a study from Spain showed that about $60.5 \%$ of the female lung cancer patients were either current or former smokers (20). In addition, the prevalence of smokers in women with lung cancer was $70-85 \%$ in another Western population studied (12). This difference in the proportions is presumably due to demographic differences. We assume that the elderly female population in Asia may be a relatively smaller group of smokers compared to other global regions, and the culture of social disapproval of women's smoking may have contributed to the difference in prevalence. Secondly, the prevalence of EGFR mutations was much higher in an Asian female population than in other regions $(13,17)$. The present study also showed that about $48 \%$ had positive EGFR mutations. This proportion is higher than that reported in a European population $(14,21)$. With respect to pathologic subtype, the ever-smoker group had a significantly higher proportion of squamous and small cell lung cancer than the never-smoker group and this difference in the pathologic types regarding smoking status was seen in previous studies, including a study in a Korean population $(11,18,20)$.

The prevalence of smoking experience in our study was about $12.5 \%$, which was relatively low when compared to European women with lung cancer (22), and it is comparable to the smoking rate among general female population of Korea (23). Looking from a different perspective, it is possible that a considerable proportion of 
Table 2 Comparison between ever and never smokers in NSCLC

\begin{tabular}{|c|c|c|c|c|}
\hline Characteristics & Total & Never smoker & Ever smoker & $P$ value \\
\hline Age (year) & $66.5 \pm 12.1$ & $66.2 \pm 12.0$ & $69.2 \pm 12.6$ & 0.048 \\
\hline BMI $\left(\mathrm{kg} / \mathrm{m}^{2}\right)$ & $24.8 \pm 20.8$ & $25.1 \pm 21.9$ & $22.5 \pm 4.2$ & 0.017 \\
\hline Initial symptoms, n (\%) & & & & 0.359 \\
\hline Cough & $208(30.5)$ & $185(30.2)$ & $23(32.9)$ & \\
\hline Sputum & $107(15.7)$ & $93(15.2)$ & $14(20.0)$ & \\
\hline Dyspnea & $133(19.5)$ & $111(18.1)$ & $22(31.4)$ & \\
\hline Hoarseness & $6(0.9)$ & $5(0.8)$ & $1(1.4)$ & \\
\hline ECOG, n (\%) & $n=506$ & $n=455$ & $n=51$ & 0.098 \\
\hline $0-2$ & $484(95.7)$ & $438(96.3)$ & $46(90.2)$ & \\
\hline $3-4$ & $22(4.3)$ & $17(3.7)$ & $5(9.8)$ & \\
\hline Median survival time (months) & $28.0[10.0-35.0]$ & $29.0[10.0-35.0]$ & $14.0[5.0-32.0]$ & 0.002 \\
\hline Clinical stage, $\mathrm{n}(\%)$ & $\mathrm{n}=676$ & $n=606$ & $\mathrm{n}=70$ & \\
\hline I & $262(38.8)$ & $244(40.3)$ & $18(25.7)$ & \\
\hline II & $44(6.5)$ & $38(6.3)$ & $6(8.6)$ & \\
\hline Number (\%) & $24(7.5)$ & $23(7.8)$ & $1(3.7)$ & \\
\hline Initial treatment, n (\%) & $n=629$ & $n=568$ & $n=61$ & \\
\hline Surgery only & 216 (34.3) & $202(35.6)$ & $14(23.0)$ & \\
\hline Surgery + adjuvant & $95(15.1)$ & $89(15.7)$ & $6(9.8)$ & \\
\hline Conventional chemotherapy & 125 (19.9) & $111(19.5)$ & $14(23.0)$ & \\
\hline CCRT & $17(2.7)$ & $14(2.5)$ & $3(4.9)$ & \\
\hline Targeted therapy & $75(11.9)$ & $73(12.9)$ & $2(3.3)$ & \\
\hline Radiotherapy only & $34(5.4)$ & $30(5.3)$ & $4(6.6)$ & \\
\hline Supportive care only & $67(10.7)$ & $49(8.6)$ & $18(29.5)$ & \\
\hline Target agent usage (ever), n (\%) & $154(22.5)$ & $147(24.0)$ & $7(10.0)$ & 0.012 \\
\hline EGFR inhibitor ever & $143(20.9)$ & $136(22.2)$ & $7(10.0)$ & 0.026 \\
\hline ALK inhibitor ever & $16(2.3)$ & $16(2.6)$ & $0(0.0)$ & 0.342 \\
\hline
\end{tabular}

Table 2 (continued) 
Table 2 (continued)

\begin{tabular}{|c|c|c|c|c|}
\hline Characteristics & Total & Never smoker & Ever smoker & $P$ value \\
\hline FVC (liter) & $2.5 \pm 0.6$ & $2.5 \pm 0.6$ & $2.3 \pm 0.7$ & 0.017 \\
\hline FEV1 (\%) & $63.2 \pm 16.7$ & $64.3 \pm 16.3$ & $53.4 \pm 17.0$ & 0.000 \\
\hline FVC (\%) & $71.9 \pm 16.2$ & $72.5 \pm 16.0$ & $66.6 \pm 16.8$ & 0.013 \\
\hline FEV1/FVC $<0.7$ & $72 / 499$ & $48 / 447$ & $24 / 52$ & 0.000 \\
\hline DLCO (absolute) & $14.5 \pm 4.3$ & $14.9 \pm 4.1$ & $11.4 \pm 4.4$ & 0.000 \\
\hline DLCO (\%) & $85.8 \pm 22.3$ & $87.7 \pm 21.6$ & $68.8 \pm 22.0$ & 0.000 \\
\hline
\end{tabular}

ALK, anaplastic lymphoma kinase; BMI, body mass index; ECOG, Eastern Cooperative Oncology Group; EGFR, epidermal growth factor receptor; FEV1, forced expiratory volume in $1 \mathrm{~s}$; FVC; forced vital capacity; DLCO, diffusing capacity of the lung for carbon monoxide.

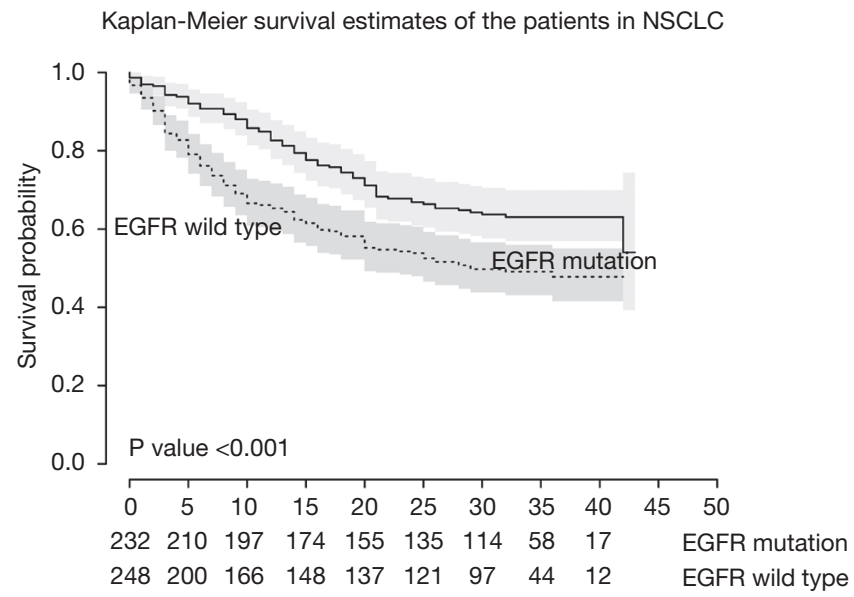

Figure 3 Kaplan-Meier survival curves for the epidermal growth factor receptor (EGFR) positive and wild-type patients with nonsmall cell lung cancer.

patients who were reported as never smoker, may have been exposed to passive smoking. In the previous study of Korean women with lung cancer (23), the passive smoking rate was $7.9 \%$ in non-smoking women. However, this survey was made in 2016, and we assume that the actual passivesmoking rate would have been higher in the past time, during which a large number of our study patients could have been exposed to. Unfortunately, no data on passive smoking were present in the current study. In addition, the smoking status was based on self-reporting, and the statistics on Korean women showed that smoking rate was about $6 \%$ which was also based on self-reporting (23), but we suspect that the actual rate is much higher. Thus, it is possible that disparity in actual number of ever smokers could be present due to the limitation of a self-reporting method.

The number of the never smoker group is about 8.5 times that of the smoker group in NSCLC, and the proportion of stage I cancer is about $40.3 \%$, which is higher than $25.7 \%$ of the smoker group. In early stage lung cancer, it is difficult for patients to be aware of presenting symptoms $(24,25)$, and the screening by simple chest X-ray is not as effective as in advanced cancer. Thus, a more effective screening tool, such as low dose chest CT may increase the chance of a curative treatment for the early stage lung cancer in the never smoker female population. Considering both the number and proportion of early stage lung cancer, our study results support the necessity of a more vigorous screening strategy using low dose CT in never smoker female population with risk factors for cancer development.

The reason why smoking experience was found to be an independent factor for shorter survival is not clear. It is possible that smoking results in decreased lung function, which further contributes to the poor prognosis of lung cancer patients. In the present study, the ever-smoker group had significantly lower FEV1, FVC, and DLCO in both the overall patient group and the NSCLC subgroup. The impact of decreased lung functions on prognosis will be discussed in a later section. A study by a French group showed that patients with positive cotinine during chemotherapy showed poorer overall response rates compared to never-smokers (26). Gemine et al. showed that after adjusting for various factors, smokers with NSCLC were more likely to die within one year compared to 
Table 3 Survival analysis in NSCLC

\begin{tabular}{|c|c|c|c|c|c|c|}
\hline Characteristics & \multicolumn{3}{|c|}{ Univariate analysis } & \multicolumn{3}{|c|}{ Multivariate analysis } \\
\hline Age & 1.04 & $1.03-1.05$ & 0.000 & 1.01 & $0.99-1.03$ & 0.373 \\
\hline BMI & 1 & $0.99-1.01$ & 0.434 & 0.96 & $0.89-1.02$ & 0.181 \\
\hline \multicolumn{7}{|l|}{ ECOG } \\
\hline $3-4$ & 6.72 & $3.99-11.31$ & 0.000 & 2.75 & $1.05-7.22$ & 0.04 \\
\hline \multicolumn{7}{|l|}{ Smoking experience } \\
\hline Never smoker & 1 & & & 1 & & \\
\hline Ever smoker & 1.95 & $1.4-2.72$ & 0.000 & 2.39 & $1.12-5.11$ & 0.025 \\
\hline Supportive treatment & 5.93 & $4.31-8.16$ & 0.000 & 3.14 & $1.22-8.06$ & 0.017 \\
\hline \multicolumn{7}{|l|}{ EGFR mutation } \\
\hline- & 1 & & & 1 & & \\
\hline+ & 0.60 & $0.45-0.8$ & 0.000 & 0.63 & $0.41-0.96$ & 0.033 \\
\hline \multicolumn{7}{|l|}{ Clinical Stage } \\
\hline $1 / I I$ & 1 & & & 1 & & \\
\hline III/IV & 15.49 & $9.99-24.02$ & 0.000 & 11.88 & $5.79-24.38$ & 0.000 \\
\hline \multicolumn{7}{|l|}{ Histopathology } \\
\hline
\end{tabular}

BMI, body mass index; ECOG, Eastern Cooperative Oncology Group; EGFR, epidermal growth factor receptor; FVC, forced vital capacity.

never-smokers (27). It is possible that smoking has more detrimental effects in female lung cancer patients compared to male patients because they have relatively smaller lung volumes than men, and therefore, tobacco smoke can damage a larger proportion of the lung. Ryu et al. showed that among Korean patients with lung cancer, the unfavorable effects of smoking on pulmonary function were greater in women compared to men and suggested that the higher susceptibility might be attributed to lower lung volume (28).

We also investigated whether pulmonary function parameters were independent factors associated with overall survival. Mean FEV1 and FVC values of the never smoker groups were relatively low, considering the absent effect of smoking. However, in case of early-stage NSCLC, mean values of FEV1 (\%) and FVC (\%) were much higher than the mean values of all never smoker patients with NSCLC. Decreased FVC (\%) was predictive of shorter survival in our study. Few studies showed that FVC (\%) predicted worse prognosis in NSCLC patients. Low FVC (\%) was shown to be associated with cytotoxic chemotherapyrelated acute exacerbation of interstitial lung disease in lung cancer (29). We assume that FVC (\%) not only reflected lung function but also lung volume indirectly. A study by Vandevoorde et al. showed that reduced FVC was highly correlated with reduced total lung capacity in female patients (30). However, further studies using plethysmography are necessary to confirm how pulmonary 
function has prognostic value in female NSCLC.

Although it was not shown in the results of the present study, each FEV1 (\%) or fixed airway obstruction defined as $\mathrm{FEV} 1 / \mathrm{FVC}<70 \%$ was entered into survival analyses in place of FVC (\%) in other Cox regression models. In NSCLC, FEV1 (\%) also showed a significant association with survival, consistent with the results of a previous study (31). However, FEV1/FVC $<70 \%$ was not an independent prognostic factor. This statistical insignificance was shown in a study by Lee et al. (32) and is contrary to the results of a previous study on patients with chronic obstructive pulmonary disease (COPD) and NSCLC (33).

There were some limitations to the present study. First, data on second-hand smoke was not obtained in this study. Considering that many never-smokers are exposed to passive smoking in their households, a future study accounting for this factor is necessary. Second, the TNM classification of the $7^{\text {th }}$ edition was used to define cancer stages in the patients, so this should be taken into account when applying the results of our study to other lung cancer populations. Third, comorbidities including interstitial lung disease were not evaluated in our study. Lastly, our data were retrospectively collected from the randomly sampled patients group representing whole newly diagnosed lung cancer population in 2014, however, not all patients with newly diagnosed lung cancer were evaluated in this study. We believe that limitation of relatively small number of study patients were overcome by careful sorting sampling process, but it should be taken into account for interpretation of results.

\section{Conclusions}

In conclusion, in women with NSCLC, smoking was associated with worse prognosis and decreased lung function was significantly associated with mortality. Further studies are necessary to clarify the underlying mechanisms of the association between smoking and unfavorable outcomes in Korean women with lung cancer.

\section{Acknowledgments}

Funding: This study was supported by the Health Promotion Fund, Ministry of Health \& Welfare, Republic of Korea (1760580-1). The data used for this study were provided by the KALC and the Ministry of Health and Welfare, KCCR.

\section{Footnote}

Reporting Checklist: The authors have completed the STROBE reporting checklist. Available at http://dx.doi. org/10.21037/jtd-20-1671

Conflicts of Interest: All authors have completed the ICMJE uniform disclosure form (available at http://dx.doi. org/10.21037/jtd-20-1671). The authors have no conflicts of interest to declare.

Ethical Statement: The authors are accountable for all aspects of the work in ensuring that questions related to the accuracy or integrity of any part of the work are appropriately investigated and resolved. The study was conducted in accordance with the Declaration of Helsinki (as revised in 2013). The study protocol was reviewed and approved by the Institutional Review Board at the National Cancer Center (NCC2018-0193), which waived the requirement for informed consent due to the retrospective nature of the study.

Open Access Statement: This is an Open Access article distributed in accordance with the Creative Commons Attribution-NonCommercial-NoDerivs 4.0 International License (CC BY-NC-ND 4.0), which permits the noncommercial replication and distribution of the article with the strict proviso that no changes or edits are made and the original work is properly cited (including links to both the formal publication through the relevant DOI and the license). See: https://creativecommons.org/licenses/by-nc-nd/4.0/.

\section{References}

1. Ferlay J, Steliarova-Foucher E, Lortet-Tieulent J, et al. Cancer incidence and mortality patterns in Europe: estimates for 40 countries in 2012. Eur J Cancer 2013;49:1374-403.

2. Torre LA, Bray F, Siegel RL, et al. Global cancer statistics, 2012. CA Cancer J Clin 2015;65:87-108.

3. Egleston BL, Meireles SI, Flieder DB, et al. Populationbased trends in lung cancer incidence in women. Semin Oncol 2009;36:506-15.

4. Pauk N, Kubik A, Zatloukal P, et al. Lung cancer in women. Lung Cancer 2005;48:1-9.

5. Kligerman S, White C. Epidemiology of lung cancer in women: risk factors, survival, and screening. AJR Am J 
Roentgenol 2011;196:287-95.

6. North CM, Christiani DC. Women and lung cancer: what is new? Semin Thorac Cardiovasc Surg 2013;25:87-94.

7. Alberg AJ, Wallace K, Silvestri GA, et al. Invited commentary: the etiology of lung cancer in men compared with women. Am J Epidemiol 2013;177:613-6.

8. Mazières J, Rouquette I, Lepage B, et al. Specificities of lung adenocarcinoma in women who have never smoked. J Thorac Oncol 2013;8:923-9.

9. Heilbroner SP, Xanthopoulos EP, Buono D, et al. Impact of estrogen monotherapy on survival in women with stage III-IV non-small cell lung cancer. Lung Cancer 2019;129:8-15.

10. Cho J, Choi SM, Lee J, et al. Proportion and clinical features of never-smokers with non-small cell lung cancer. Chin J Cancer 2017;36:20.

11. Zeng Q, Vogtmann E, Jia MM, et al. Tobacco smoking and trends in histological subtypes of female lung cancer at the Cancer Hospital of the Chinese Academy of Medical Sciences over 13 years. Thorac Cancer 2019;10:1717-24.

12. Parkin DM, Bray F, Ferlay J, et al. Global cancer statistics, 2002. CA Cancer J Clin 2005;55:74-108.

13. Shi Y, Au JS, Thongprasert S, et al. A prospective, molecular epidemiology study of EGFR mutations in Asian patients with advanced non-small-cell lung cancer of adenocarcinoma histology (PIONEER). J Thorac Oncol 2014;9:154-62.

14. Dearden S, Stevens J, Wu YL, et al. Mutation incidence and coincidence in non small-cell lung cancer: metaanalyses by ethnicity and histology (mutMap). Ann Oncol 2013;24:2371-6.

15. Korea S. Annual prevalence of newly diagnosed lung cancer. Daejeon: Statistics Korea. Available online: http:// kosiskr/statHtml/statHtmldo?orgId=117\&tblId=DT_11 7N_A00023\&conn_path=I2

16. Mitsudomi T, Kosaka T, Endoh H, et al. Mutations of the epidermal growth factor receptor gene predict prolonged survival after gefitinib treatment in patients with nonsmall-cell lung cancer with postoperative recurrence. J Clin Oncol 2005;23:2513-20.

17. Shigematsu H, Lin L, Takahashi T, et al. Clinical and biological features associated with epidermal growth factor receptor gene mutations in lung cancers. J Natl Cancer Inst 2005;97:339-46.

18. Choi CM, Kim HC, Jung CY, et al. Report of the Korean Association of Lung Cancer Registry (KALC-R), 2014. Cancer Res Treat 2019;51:1400-10.
19. Choi JK PD, Lee JO. Normal Predictive values of spirometry in Korean Population. Tuberc Respir Dis (Seoul) 2005;58:230-42.

20. Viñolas N, Garrido P, Isla D, et al. Lung Cancer in Never-Smoking Women: A Sub-Analysis of the Spanish Female-Specific Database WORLD07. Cancer Invest 2017;35:358-65.

21. Rosell R, Moran T, Queralt C, et al. Screening for epidermal growth factor receptor mutations in lung cancer. N Engl J Med 2009;361:958-67.

22. Salmerón D, Chirlaque MD, Isabel Izarzugaza M, et al. Lung cancer prognosis in Spain: the role of histology, age and sex. Respir Med 2012;106:1301-8.

23. Park CK, Kim SJ. Trends and Updated Statistics of Lung Cancer in Korea. Tuberc Respir Dis (Seoul) 2019;82:175-7.

24. Quaife SL, Forbes LJ, Ramirez AJ, et al. Recognition of cancer warning signs and anticipated delay in help-seeking in a population sample of adults in the UK. Br J Cancer 2014;110:12-8.

25. Simon AE, Juszczyk D, Smyth N, et al. Knowledge of lung cancer symptoms and risk factors in the U.K.: development of a measure and results from a population-based survey. Thorax 2012;67:426-32.

26. Dacosta-Noble P, Costantini A, Dumenil C, et al. Positive plasma cotinine during platinum-based chemotherapy is associated with poor response rate in advanced non-small cell lung cancer patients. PLoS One 2019;14:e0219080.

27. Gemine RE, Ghosal R, Collier G, et al. Longitudinal study to assess impact of smoking at diagnosis and quitting on 1-year survival for people with non-small cell lung cancer. Lung Cancer 2019;129:1-7.

28. Ryu JS, Jeon SH, Kim JS, et al. Gender differences in susceptibility to smoking among patients with lung cancer. Korean J Intern Med 2011;26:427-31.

29. Enomoto Y, Inui N, Kato T, et al. Low forced vital capacity predicts cytotoxic chemotherapy-associated acute exacerbation of interstitial lung disease in patients with lung cancer. Lung Cancer 2016;96:63-7.

30. Vandevoorde J, Verbanck S, Schuermans D, et al. Forced vital capacity and forced expiratory volume in six seconds as predictors of reduced total lung capacity. Eur Respir J 2008;31:391-5.

31. Lee JH, Song EM, Sim YS, et al. Forced expiratory volume in one second as a prognostic factor in advanced non-small cell lung cancer. J Thorac Oncol 2011;6:305-9.

32. Lee SJ, Lee J, Park YS, et al. Impact of chronic obstructive 
pulmonary disease on the mortality of patients with nonsmall-cell lung cancer. J Thorac Oncol 2014;9:812-7.

33. Lim JU, Yeo CD, Rhee CK, et al. Comparison of clinical characteristics and overall survival between spirometrically diagnosed chronic obstructive pulmonary disease (COPD) and non-COPD never-smoking stage I-IV non-small cell lung cancer patients. Int J Chron Obstruct Pulmon Dis 2019;14:929-38.

Cite this article as: Lim JU, Han S, Kim HC, Choi CM, Jung CY, Cho DG, Jeon JH, Lee JE, Ahn JS, Kim Y, Choi YD, Suh YG, Kim JE, Won YJ, Kim YC, Park CK, Kim SJ. Characteristics of female lung cancer in Korea: analysis of Korean National Lung Cancer Registry. J Thorac Dis 2020;12(9):4612-4622. doi: 10.21037/jtd-20-1671 


\section{Supplementary}

Table S1 Comparison between EGFR mutation vs. EGFR wild type subgroups in NSCLC

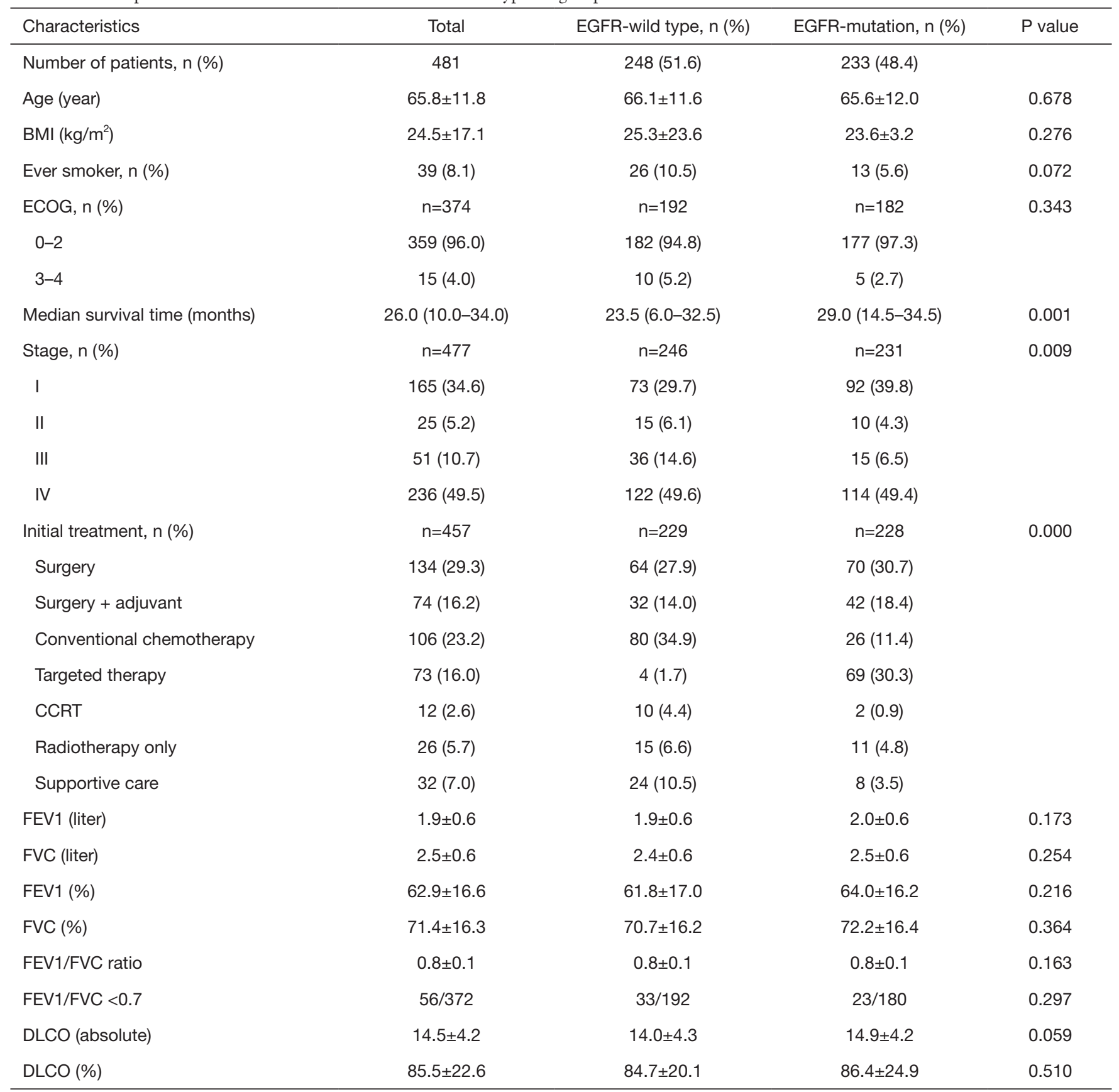

EGFR, epidermal growth factor receptor; NSCLC, non-small cell lung cancer; BMI, body mass index; ECOG, Eastern Cooperative Oncology Group; FEV1, forced expiratory volume in $1 \mathrm{~s}$; FVC, forced vital capacity; DLCO, diffusing capacity of the lung for carbon monoxide. 